\title{
Editorials
}

\section{Bereavement care during and after the COVID-19 pandemic}

\section{INTRODUCTION}

As the world navigates the ongoing challenges of the COVID-19 pandemic, bereavement care has come to the fore with renewed significance.

The scale of the impact on bereavement is now emerging: for every COVID-19 death it is estimated that up to nine people are affected by bereavement, ${ }^{1}$ and those bereaved are likely to display higher levels of prolonged grief symptoms. ${ }^{2}$ For all people bereaved during this period, whether from COVID-19, other conditions, or deaths prior to the pandemic, there are multiple risk factors for complex grieving: an increase in sudden and unexpected deaths; restrictions on visiting family members at the end of life; disruption to mourning practices and funerals; and reduced access to social support networks. ${ }^{3,4}$

Healthcare professionals, including GPs and other primary care practitioners, have also faced multiple challenges during this period in supporting bereaved people; adapting to remote technology, managing the increased complexities of bereaved relatives' grief, and dealing with their own professional and personal experiences of bereavement. ${ }^{4}$

General practice has a key role in addressing this 'tsunami of grief'.4 Comprehensive bereavement care to alleviate the impact on the many people who have been bereaved is vital: bereavement is an important cause of mortality and morbidity, impacting on physical and mental health, and leading to increased use of health services. ${ }^{5}$ It is timely to address the role of primary care in bereavement care, and to ask how general practice can better support bereaved people, and how practitioners can themselves be better supported in caring for bereaved people during and after the pandemic.

while bereavement is considered an important part of primary care, provision is variable and clinicians are often unsure of how much to become involved with bereaved patients.

\section{BEREAVEMENT CARE IN GENERAL PRACTICE}

Over 20 years ago, Richard Woof expressed the view that while UK GPs would continue to provide useful support to bereaved people, comprehensive introduction of bereavement care into primary care practice was unlikely. Such views remain salient today despite the substantial developments that have taken place both in bereavement research and the organisation of general practice.

For many bereaved people, family, friends, and wider community networks are sufficient support as they adjust to their painful loss. Many also value bereavement support from the range of available community, charitable, and voluntary organisations, including GPs and their wider primary care team members, bereavement counsellors, social care workers, and palliative care teams.

Our recent realist review of primary care management of bereavement and complicated grief ${ }^{7}$ found that while bereavement is considered an important part of primary care, provision is variable and clinicians are often unsure of how much to become involved with bereaved patients. Practitioners report feeling unprepared to manage the personal emotional challenges of supporting distressed grieving patients. There is variable awareness of the severe, complicated, and prolonged grief symptoms that affect a minority of bereaved. Identification of bereaved people is challenging as patient deaths are rarely recorded in the records of

\section{"Supporting bereaved people requires a multiprofessional and community response that includes general practice, primary care professionals, informal caring networks, and bereavement care providers.}

their bereaved relatives, who may be patients of a different practice.

\section{THE PERSONAL AND THE PRIVATE}

Underlying Woof's view was the question of whether an expansion of bereavement support in primary care was desirable. In the UK, the professionalisation of death and dying, and the fragmentation of social and community ties has meant that for those seeking compassionate support, the GP is often the first 'port of call'. Bereaved patients report wanting and expecting acknowledgement from their GP following bereavement; a period where they often feel overwhelmed and do not know where to turn for support. However, our review suggests that GPs are at times apprehensive about proactively engaging with bereaved patients due to concerns of medicalising grief or intruding into a private life event.?

Clinicians are often concerned at the lack of time and primary care team resources available to engage with bereaved patients. Our realist analysis indicates that bereavement care is viewed as demanding not only on time, but also on the emotional resources of GPs and nurses. ' With no 'treatment' available for the pain of bereavement beyond active and supportive listening, grieving patients may instil feelings of powerlessness in clinicians.

Furthermore, limited education concerning bereavement and training in bereavement care at times leads practitioners to refer to their own experiences of bereavement to foster empathy with patients. While this might increase confidence, it can also make encounters with patients more difficult, by resurfacing practitioners' own feelings of grief. As a result, practitioners may require support to appropriately negotiate their personal and professional boundaries with bereaved patients.

Supporting bereaved people requires a multiprofessional and community response 
that includes general practice, primary care professionals, informal caring networks, and bereavement care providers. For GPs under significant time and workload pressures, social prescribing and link workers may help connect patients with local support services or community groups, allowing GPs to focus their attention to those with more severe grief symptoms who may need medical attention. Primary care networks could helpfully develop resources to signpost practice teams and their bereaved patients to local and national resources, and support services.

\section{RELATIONSHIP-BASED CARE}

While significant knowledge gaps concerning effective bereavement interventions remain, GPs already possess many skills required to support bereaved patients. A renewed focus on relationship-based care in general practice bodes well for improving care of bereaved patients. ${ }^{8-10}$ Both GPs and patients identify the trust and established relationships that come with continuity of care, with associated understanding of patient preferences and needs, as beneficial in knowing how to best support patients following bereavement. ${ }^{7}$ Awareness of, and ability to conduct risk assessments of, bereaved patients is important, but a broader understanding of patient needs that comes with an existing doctor-patient relationship may be more appropriate for people who primarily seek acknowledgement of their bereavement and a clinician with supportive communication and listening skills.

As lona Heath noted in a recent BJGP editorial, ${ }^{9}$ general practice involves the transactional and relational: the transactional and technical skills of GPs have a limited role in the support of those struggling with grief, while a strong one-to-one relationship of mutual respect and trust can be immensely powerful.

The pandemic has challenged the ability of clinicians to sustain longitudinal and supportive relationships with patients due to necessary social distancing measures of personal protective equipment (PPE) and remote consultations. ${ }^{4}$ As we begin to emerge from the pandemic, such challenges offer opportunities to rediscover the power of therapeutic relationships and re-emphasise the central importance of relational care in general practice. ${ }^{11}$ Trusting relationships and compassionate communication between clinicians and bereaved families are critical to bereavement care, if challenging to ensure through remote and online routes, creating additional burdens of time and emotional resources for an already overstretched and exhausted workforce. Additional resources of finance and personnel are needed for primary care, to ensure that the relationshipbased and compassionate care, so crucial to bereavement care and many other aspects of general practice, is sustained and developed. This may be particularly challenging for trainee and newly qualified GPs who are grappling with the additional challenges of learning and developing relationships with patients in the context of the pandemic. The impacts of the planned reorganisation of primary care on such aspirations remains unclear.12

\section{CONCLUSION}

Bereavement care remains a central and important aspect of general practice. As primary care emerges from the pandemic with radically altered ways of working. renewed emphasis on relationships and continuity of care, combined with addressing the persistent gaps in bereavement care education, resources and skills, together with adequate funding of primary care services, will ensure that neither practitioners nor patients are left to suffer the burden of bereavement alone.

\section{Caroline Pearce,}

Research Associate, Primary Care Unit, Department of Public Health and Primary Care, University of Cambridge, Cambridge.

\section{Geoff Wong,}

GP and Associate Professor, Nuffield Department of Primary Care Health Sciences, University of Oxford, Oxford.

\section{Stephen Barclay,}

GP and University Senior Lecturer, Primary Care Unit, Department of Public Health and Primary Care, University of Cambridge, Cambridge.

\section{Funding}

All authors' salaries are partly supported by The Evidence Synthesis Working Group of the National Institute for Health Research School for Primary Care Research (NIHR SPCR) (project number: 390). Stephen Barclay is supported by the NIHR Applied Research Collaboration East of England programme. The views expressed are those of the author(s) and not necessarily those of the NIHR, the NHS, or the Department of Health and Social Care.

\section{Provenance}

Commissioned; externally peer reviewed.

\section{Competing interests}

Geoff Wong is Deputy Chair of the United Kingdom's National Institute for Health Research Health Technology Assessment Prioritisation Committee: Integrated Community Health and Social Care (A), and member of the Methods Group (A).

DOI: https://doi.org/10.3399/bjgp21X715625

\section{ADDRESS FOR CORRESPONDENCE}

\section{Caroline Pearce}

Primary Care Unit, Department of Public Health and Primary Care, Forvie Site, University of Cambridge, Cambridge CB2 OSR, UK.

Email: cmp89amedschl.cam.ac.uk

\section{REFERENCES}

1. Verdery AM, Smith-Greenaway E, Margolis R, Daw J. Tracking the reach of COVID-19 kin loss with a bereavement multiplier applied to the United States. Proc Natl Acad Sci U S A 2020; 117(30): 17695-17701.

2. Eisma MC, Tamminga A, Smid GE, Boelen PA. Acute grief after deaths due to COVID-19, natural causes and unnatural causes: an empirical comparison. J Affect Disord 2020; 278: 54-56.

3. Harrop E, Farnell D, Longo M, et al. Supporting people bereaved during COVID-19: study report 1. 2020. https:// www.covidbereavement.com/reports-andpublications (accessed 6 Apr 2021).

4. Pearce C, Honey JR, Lovick R, et al. 'A silent epidemic of grief': a survey of bereavement care provision in the UK and Ireland during the COVID-19 pandemic. BMJ Open 2021; 11(3): e046872.

5. King $M$, Vasanthan $M$, Petersen I, et al. Mortality and medical care after bereavement: a general practice cohort study. PLoS One 2013; 8(1): e52561.

6. Woof WR. The future of bereavement care in British general practice. Eur J Cancer Care (Engl) 1997; 6(2): 133-136.

7. Pearce C, Wong G, Kuhn I, Barclay S. Supporting bereavement and complicated grief in primary care: a realist review. BJGP Open 2021; DOI: https://doi.org/10.3399/ BJGPO.2021.0008.

8. Royal College of General Practitioners. Policy and campaigning priorities 2020-23. https://www.rcgp.org.uk/policy/policyand-campaigning-priorities-2020-23.aspx laccessed 6 Apr 2021).

9. Heath I. COVID-19 and the legacy of grief. $\mathrm{Br} J$ Gen Pract 2020; DOI: https://doi.org/10.3399/ bjgp20X712181.

10. Jones R. General practice in the years ahead: relationships will matter more than ever. $\mathrm{Br} \mathrm{J}$ Gen Pract 2020; DOI: https://doi.org/10.3399/ bjgp21X714341.

11. Pereira Gray D, Freeman G, Johns C, Roland M. Covid 19: a fork in the road for general practice. BMJ 2020; 370: m3709.

12. Roderick P. Pollock AM. A new bill to reform the NHS in England: the wrong proposals at the wrong time. BMJ Opinion 2021; 11 Feb: https://blogs.bmj.com/bmj/2021/02/11/anew-bill-to-reform-the-nhs-in-englandthe-wrong-proposals-at-the-wrong-time (accessed 6 Apr 2021). 